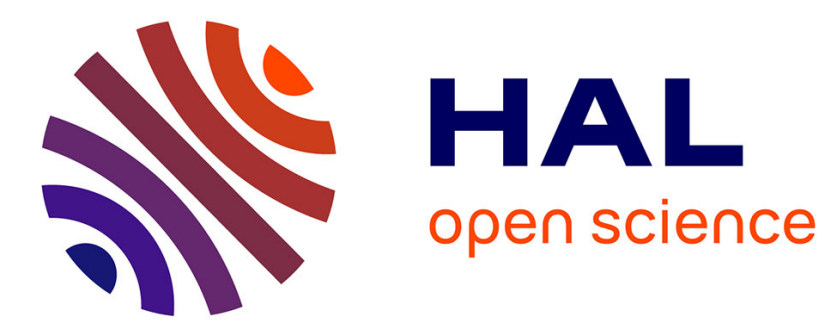

\title{
Partitioned schemes of the finite-element method for dynamic problems of acoustoelectroelasticity
}

\author{
A. V. Belokon', V. A. Eremeyev, A. V. Nasedkin, A. N. Solov'Yev
}

\section{To cite this version:}

A. V. Belokon', V. A. Eremeyev, A. V. Nasedkin, A. N. Solov'Yev. Partitioned schemes of the finiteelement method for dynamic problems of acoustoelectroelasticity. Journal of Applied Mathematics and Mechanics, 2000, 64 (3), pp.367-377. hal-00835595

\section{HAL Id: hal-00835595 \\ https://hal.science/hal-00835595}

Submitted on 19 Jun 2013

HAL is a multi-disciplinary open access archive for the deposit and dissemination of scientific research documents, whether they are published or not. The documents may come from teaching and research institutions in France or abroad, or from public or private research centers.
L'archive ouverte pluridisciplinaire HAL, est destinée au dépôt et à la diffusion de documents scientifiques de niveau recherche, publiés ou non, émanant des établissements d'enseignement et de recherche français ou étrangers, des laboratoires publics ou privés. 


\title{
PARTITIONED SCHEMES OF THE FINITE-ELEMENT METHOD FOR DYNAMIC PROBLEMS OF ACOUSTOELECTROELASTICITY
}

\author{
A. V. BELOKON', V. A. EREMEYEV, A. V. NASEDKIN \\ and A. N. SOLOV'YEV \\ Rostov-on-Don \\ e-mail: nasedkin@math.rsu.ru
}

\begin{abstract}
A number of schemes which use partitioned forms of the matrices of the finite-element method are proposed for dynamic problems of acoustoelectric elasticity. The features of the solution, specific for electroelasticity, of generalized eigenvalue problems for large sparse matrices, and also of the realization of the method of expansion in eigenforms in harmonic nonstationary problems are pointed out. An explicit-implicit scheme for the integration with respect to time of the equations of the finite-element method of non-stationary acoustoelectroelasticity is proposed and discussed. Approaches to analysing pyroelectric devices at low modulation frequencies are indicated. The ARPACK software packages for solving eigenvalue problems are integrated into the ACELAN specialized finite-element system, for which corresponding partitioned algorithms are realized. Numerical experiments are presented on determining the first eigenfrequencies and forms of the oscillations of an axisymmetric composite piezoelectric radiator in ACELAN and in the well-known ANSYS finite-element software package. The method of expansion in eigenforms in illustrated by the analysis of a longitudinal-longitudinal piezoelectric transformer. (C) 2000 Elsevier Science Ltd. All rights reserved.
\end{abstract}

A characteristic feature of dynamic problems of acoustoelectroelasticity is the connectedness of the different kinds of physical-mechanical fields. When designing piezoelectric devices, because of the presence of a piezoelectric effect, it is necessary to take the connectedness of the mechanical and electric fields into account. When piezoelectric materials interact with an acoustic medium, or there are considerable changes in temperature, it is also necessary to take into account the acoustic or temperature fields. In general, in the case of a partial finite-element approximation of linear problems of acoustothermoelectroelasticity (with respect to the space coordinates), by combining known results $[1,2]$ one can obtain vector equations of the form

$$
\mathbf{M} \cdot \ddot{i}+\mathbf{C} \cdot \mathbf{a}+\mathbf{K} \cdot \mathbf{a}=\mathbf{F}
$$

where

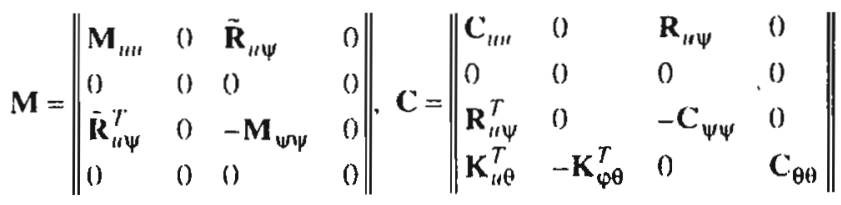

$$
\begin{aligned}
& \mathbf{K}=\left\|\begin{array}{llll}
\mathbf{K}_{u \prime \prime} & \mathbf{K}_{u \varphi} & 0 & -\mathbf{K}_{u \theta \theta} \\
\mathbf{K}_{u \varphi}^{T} & -\mathbf{K}_{\varphi \varphi \rho} & 0 & \mathbf{K}_{\varphi \theta} \\
0 & 0 & -\mathbf{K}_{\psi \psi} & 0 \\
0 & 0 & 0 & \mathbf{K}_{\theta \theta}
\end{array}\right\|, \mathbf{F}=\left\{\begin{array}{l}
\mathbf{F}_{u} \\
\mathbf{F}_{\varphi} \\
0 \\
\mathbf{F}_{\theta}
\end{array}\right\} . \quad \boldsymbol{a}=\left\{\begin{array}{l}
\mathbf{U} \\
\mathbf{\Phi} \\
\mathbf{\Psi} \\
\mathbf{T}
\end{array}\right\}
\end{aligned}
$$

and for non-stationary problems one must add the initial conditions to (0.1).

Here the unknown vector of the nodal degrees of freedom $\mathbf{a}=\mathbf{a}(t)$ may contain nodal mechanical displacements $\mathbf{U}$, electric potentials $\boldsymbol{\Phi}$, the velocity potentials of the acoustic medium $\boldsymbol{\Psi}$ and the increments of the temperatures $\mathbf{T}$. Blocks of matrices $\mathbf{M}, \mathbf{C}$ and $\mathbf{K}$ and of the vector $\mathbf{F}$ are formed due to interaction of the fields, which will be indicated by subscripts. The notation in the subscripts corresponds to the following field functions: $u$ is the field of mechanical displacements, $\varphi$ is the electric potential field, $\psi$ is the velocity field potential of the acoustic medium and $\theta$ is the temperature increment or simply the temperature. 
For problems of harmonic oscillations with a frequency $f$

$$
\mathbf{a}=\overline{\mathbf{a}}(\mathbf{x}) \exp (j(\omega) /), \mathbf{F}=\tilde{\mathbf{F}}(\mathbf{x}) \exp (j(\omega)), \omega=2 \pi f
$$

from (0.1), omitting the factor $\exp (j \omega t)$ and the tilde for the amplitude values of $\widetilde{a}$ and $\widetilde{\mathrm{F}}$, we have

$$
-(1)^{2} \mathbf{M} \cdot \mathbf{a}+j(0) \mathbf{C} \cdot \mathbf{a}+\mathbf{K} \cdot \mathbf{a}=\mathbf{F}
$$

In (0.1)-(0.5) the structure of the equations of the finite-element method of problems of acoustothermoelectroelasticity, is represented in general form. In practice the storage formats of the matrices and the vectors of the finite-element method depend on the algorithms for solving problems $(0.1)$ or $(0.5)$ and the strategy for forming the finite-element objects and the operations carried out on them.

Note also the following facts:

1. there is a considerable number of zero blocks in the matrices from $(0.2)$ and $(0.3)$;

2. the degrees of freedom of the electric potential $\Phi$ are mass-free;

3. the matrices $\mathbf{R}_{u \psi}$ and $\widetilde{\mathbf{R}}_{u \psi}$ from (0.2) are extremely sparse, and they describe the interaction of the solid-state structure and the acoustic medium only at their common boundary;

4. the connectedness of the temperature field is such that, in the majority of problems requiring the temperature to be taken into account, we can neglect the blocks $\mathrm{K}_{u \theta}^{T}$ and $\mathrm{K}_{\varphi \theta}^{T}$ in matrix $\mathbf{C}$ from $(0.2)$.

As a result, for different problems, different forms of representation of the finite-element objects in (0.1)-(0.5) turn out to be convenient:

1. a completely "mixed" form, when components of different degrees of freedom are written in succession for each node in the vector a;

2. a partitioned form, when $\mathbf{U}, \boldsymbol{\Phi}, \boldsymbol{\Psi}, \mathbf{T}$ and the corresponding blocks of the matrices from (0.2) and (0.3) are formed separately;

3. a partially "mixed" form, when subvectors are distinguished individually in the vector a only for certain types of fields.

Below we will analyse the last two cases, in which it is convenient to work with partitioned matrices or when individual blocks are separated.

\section{DETERMINATION OF THE EIGENFREQUENCIES OF PIEZOELECTRIC DEVICES}

The most important characteristics of piezoelectric devices are their electric resonance frequencies $f_{r i}=\omega_{r i} /(2 \pi)$ and antiresonance frequencies $f_{a i}=\omega_{\alpha i} /(2 \pi)$. The electric resonance and antiresonance frequencies are the eigenfrequencies of the piezoelectric devices and are calculated ignoring the effect of damping, temperature factors and interactions with acoustic media. Hence, these frequencies can be found using the finite-element method from the solution of the generalized problem obtained from (0.5) using eigenvalues of the form

$$
\mathbf{K} \cdot \mathbf{a}=\omega^{2} \mathbf{M} \cdot \mathbf{a}
$$

where

$$
\mathbf{M}=\left\|\begin{array}{ll}
\mathbf{M}_{u \prime \prime} & 0 \\
0 & 0
\end{array}\right\|, \mathbf{K}=\left\|\begin{array}{ll}
\mathbf{K}_{u t \prime} & \mathbf{K}_{u \varphi} \\
\mathbf{K}_{u \varphi}^{T} & -\mathbf{K}_{\varphi \varphi}
\end{array}\right\|, \mathbf{a}=\left\{\begin{array}{l}
\mathbf{U} \\
\boldsymbol{\Phi}
\end{array}\right\}
$$

Here, the matrices $\mathbf{K}_{u \varphi}$ and $\mathbf{K}_{\varphi \varphi}$ in (1.2) differ for electric resonances and antiresonances. Mathematically this difference consists of the following. If the matrix $\mathbf{K}$ and the vector $\boldsymbol{\Phi}$ for the electric resonance frequencies are represented in the partitioned form

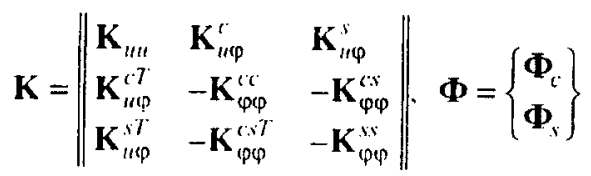

the matrix $\mathbf{K}$ and the vector $\boldsymbol{\Phi}$ for the electric antiresonance frequencies will have the form

$$
\mathbf{K}=\left\|\begin{array}{ll}
\mathbf{K}_{u \prime \prime} & \mathbf{K}_{u \varphi}^{c} \\
\mathbf{K}_{u \varphi \varphi}^{c r} & -\mathbf{K}_{\varphi \varphi}^{c l}
\end{array}\right\|, \boldsymbol{\Phi}=\boldsymbol{\Phi}_{r}
$$


Here, in the vector $\boldsymbol{\Phi}$ in (1.3) we distinguish the degrees of freedom $\boldsymbol{\Phi}_{s}$ individually for which, when finding the electric antiresonance frequencies, we assume that the electric charges at the corresponding nodes are equal to zero, and when finding the electric resonance frequencies $\Phi_{s}=0$.

Hence, to determine the electric resonance and antiresonance frequencies we need to solve problems (1.1), (1.2) twice with matrices $\mathbf{K}$ in the forms (1.3) and (1.4).

A mathematical investigation of the continual forms of eigenvalue problems (1.1)-(1.3) and (1.1), (1.2), (1.4) was carried out previously in [3]. The results obtained transfer naturally to problems discretized using the finite-element method. Thus, we have the following properties.

The matrices $\mathbf{M}$ and $\mathbf{K}$ are symmetrical; $\mathbf{M}_{u u}>0 ; \mathbf{K} \geqslant 0 ; \mathbf{K}_{u u} \geqslant 0 ; \mathbf{K}_{\varphi \varphi}>0 ; \mathbf{K}_{\varphi \varphi}^{c c}>0 ; \mathbf{K}_{\varphi \varphi}^{s s}>0$, where here and below the inequalities $\mathrm{A}>\mathrm{B}$ and $\mathrm{A} \geqslant \mathrm{B}$ for the matrices denote that the matrix $(\mathbf{A}-\mathrm{B})$ is positive definite or semi-definite respectively. In this case we assume that at least one constraint is imposed on the electric-potential field $\varphi$ which eliminates its indefiniteness apart from an arbitrary constant.

Problems (1.1)-(1.3) or (1.1), (1.2), (1.4) can be represented in the form

$$
\overline{\mathbf{K}}_{w u} \cdot \mathbf{U}=\lambda \mathbf{M}_{w a} \cdot \mathbf{U}, \lambda=\omega^{2}
$$

where

$$
\overline{\mathbf{K}}_{t m}=\mathbf{K}_{m !}+\mathbf{K}_{u \varphi} \cdot \mathbf{K}_{\varphi \varphi}^{-1} \cdot \mathbf{K}_{t w \varphi}^{T}, \boldsymbol{\Phi}=\mathbf{K}_{\varphi \varphi}^{-1} \cdot \mathbf{K}_{u \varphi}^{T} \cdot \mathbf{U}
$$

The following inequalities are satisfied for the matrices $\overline{\mathbf{K}}_{u u}$ of the problems of finding the electric antiresonance frequencies $\overline{\mathbf{K}}_{u u}^{a}$ and the resonance frequencies $\overline{\mathbf{K}}_{u u}^{a} \overline{\mathbf{K}}_{u u}^{r} \geqslant 0, \overline{\mathbf{K}}_{u u}^{r}$ where $\overline{\mathbf{K}}_{u u}^{a}$ and $\overline{\mathbf{K}}_{u u}^{r}$ are obtained from (1.6), (1.3) and (1.6), (1.4), respectively.

The eigenvalues $\lambda_{r k}=\omega_{r k}^{2}$ and $\lambda_{a k}=\omega_{a k}^{2}\left(k=1,2, \ldots, n ; n\right.$ is the order of the matrices $\mathbf{M}_{u u}$ and $\left.\mathbf{K}_{u u}\right)$ are real and non-negative. The eigenvectors corresponding to them, which we will denote by $\mathbf{W}_{r k}$ and $\mathbf{W}_{a k}$, form bases in $R^{n}$, where these vectors can be chosen to be orthonormalized with respect to the matrices of mass $\mathbf{M}_{u u}$

$$
\begin{gathered}
\left\langle\mathbf{W}_{\gamma k}, \mathbf{W}_{\gamma m}\right\rangle=\mathbf{W}_{\gamma k}^{T} \cdot \mathbf{M}_{m !} \cdot \mathbf{W}_{\gamma m}=\delta_{k m} \\
\mathbf{W}_{\gamma k}^{T} \cdot \overline{\mathbf{K}}_{m ! m}^{\gamma} \cdot \mathbf{W}_{\gamma m}=\omega_{\gamma m}^{2} \delta_{k m}, \quad \gamma=r, a
\end{gathered}
$$

If the electric resonance frequencies $\omega_{r k}$ and antiresonance frequencies $\omega_{a k}$ are numbered in increasing order, we have the inequalities

$$
\omega_{r k}^{2} \leqslant \omega_{a k}^{2} ; k=1,2, \ldots, n
$$

Thus, the coupled eigenvalue problems (1.1), (1.2) with respect to the triple of unknowns $\{\omega, \mathbf{U}, \boldsymbol{\Phi}\}$ are in fact the generalized eigenvalue problems (1.5), (1.6) with respect to the pairs $\{\omega, \mathbf{U}\}$. Changing from (1.1), (1.2) to problem (1.5), (1.6) we can discuss the procedure of static condensation by eliminating the degrees of freedom of $\boldsymbol{\Phi}$. If, when realizing this procedure in practice, we form the matrix $\mathbf{K}_{u u}$ explicitly, the properties of the sparseness of the matrices of the finite-element method will be lost. With such a strategy for solving generalized eigenvalue problems we can use methods which transform the matrix $\mathbf{K}_{u u}$. It is this approach for determining the natural frequencies of piezoelectric devices that is assumed in the well-known ANSYS finite-element software package [4], where the HauseholderBisection-Inverse iteration (HBI) method is used.

The change from sparse matrices to filled matrices can hardly be regarded as a successful solution. Algorithms which use partitioned forms of the matrices and which preserve the sparseness structure are more attractive.

We will show that, to solve eigenvalue problems (1.5), (1.6), one can effectively employ modern methods of solving generalized eigenvalue problems for large sparse matrices. For specific problems these methods require the user to employ procedures of multiplying a matrix by a vector, the addition of matrices and the solution of a system of linear algebraic equations.

Depending on the method employed, and also on the problems of searching for groups of eigenvalues with some extremality properties, eigenvalue problem (1.5) can be modified to one of the following fundamental forms 


$$
\begin{aligned}
& \text { A) } \mathbf{A}_{M} \cdot \mathbf{U}=\mu \mathbf{U}, \quad \mathbf{A}_{M}=\mathbf{M}_{u l l}^{-1} \cdot \mathbf{A}_{\sigma} \\
& \left.A_{s}\right) \cdot \mathbf{A}_{s} \cdot \mathbf{y}=\mu \mathbf{y}, \quad \mathbf{A}_{s}=\mathbf{L}_{M}^{-1} \cdot \mathbf{A}_{\sigma} \cdot \mathbf{L}_{M}^{-T} \\
& \text { B) } \mathbf{A}_{M}^{-1} \cdot \mathbf{U}=v \mathbf{U}, \quad \mathbf{A}_{M}^{-1}=\mathbf{A}_{\sigma}^{-1} \cdot \mathbf{M}_{w} \\
& \text { B, } \mathbf{A}_{s}^{-1} \cdot \mathbf{y}=v \mathbf{y}, \quad \mathbf{A}_{s}^{-1}=\mathbf{L}_{M}^{T} \cdot \mathbf{A}_{\sigma}^{-1} \cdot \mathbf{L}_{M}
\end{aligned}
$$

where

$$
\mathbf{y}=\mathbf{L}_{M}^{T} \cdot \mathbf{U}, \quad \mathbf{M}_{u t \prime}=\mathbf{L}_{M} \cdot \mathbf{L}_{M}^{T}, \mathbf{A}_{\sigma}=\overline{\mathbf{K}}_{u t}-\sigma \mathbf{M}_{t u t}
$$

$\mathbf{L}_{M}$ is the Cholesky factor of the matrix $\mathbf{M}_{u u}, \sigma$ is the value of the shear and $\mu=\lambda-\sigma$, $\nu=(\lambda-\sigma)$.

The matrices $\mathbf{A}_{s}$ and $\mathbf{A}_{s}^{-1}$ of problems $A_{s}$ and $B_{\mathrm{s}}$ are symmetrical, while the methods of solving these problems maintain the usual orthogonality of the calculated eigenvectors $\mathbf{y}$. The eigenvectors $\mathbf{U}=\mathbf{W}_{\gamma_{k}}$ obtained from (1.13) then turn out to be orthogonal in the sense of the scalar product from (1.7), i.e. orthogonal with respect to the matrix of masses.

The matrices $\mathbf{A}_{\mathrm{M}}$ and $\mathbf{A}_{M}^{-1}$ of problems $A$ and $B$ can be regarded as symmetrical operators in $R^{n}$ space with a scalar product from (1.7)

$$
\left\langle\mathbf{A}_{M}^{(-1)} \cdot \mathbf{a}, \mathbf{b}\right\rangle=\left\langle\mathbf{a}, \mathbf{A}_{M}^{(-1)} \cdot \mathbf{b}\right\rangle, \quad \forall \mathbf{a}, \mathbf{b} \in R^{\prime \prime}
$$

Hence, for problems $A$ and $B$ the orthogonality of the vectors $\mathbf{U}$ is usually maintained in the sense of the scalar product from (1.7).

Hence, problems $A$ and $A_{s}$ or $B$ and $B_{s}$ and methods for solving them in fact differ solely by the scalar product employed. Hence, we will only consider problems $A$ and $B$ below.

To solve problem $A$ by iterational methods for large sparse matrices we need to carry out the procedure of multiplying matrix $\mathbf{A}_{\mathbf{M}}$ by the iterated vector $\mathbf{U}$ and multiplying the matrix $\mathbf{M}_{u u}$ by a vector to calculate the scalar product from (1.7).

To do this at the preparatory stage we must form the matrix

$$
\mathbf{A}_{\sigma m}=\mathbf{K}_{m !}-\sigma \mathbf{M}_{m !}
$$

and obtain Cholesky factors $\mathbf{M}_{u u}$ and $\mathbf{K} \varphi \varphi\left(\mathbf{K}_{\varphi \varphi}=\mathbf{L}_{\varphi \varphi} \cdot \mathbf{L}_{\varphi \varphi}^{T}\right)$

$$
\mathbf{M}_{i \prime \prime} \rightarrow \mathbf{L}_{M}, \mathbf{K}_{\varphi \varphi} \rightarrow \mathbf{L}_{\varphi \varphi}
$$

Further, the algorithm for calculating $z=\mathbf{A}_{M} \cdot \mathbf{U}$ for partitioned matrices, as follows from (1.9), (1.6), (1.14) and (1.15), can be realized as follows:

$$
\begin{gathered}
\mathbf{x}_{1}:=\mathbf{K}_{\mu \varphi}^{T} \cdot \mathbf{U} \\
\text { Solve } \boldsymbol{L}_{\varphi \varphi} \cdot \mathbf{x}_{2}=\mathbf{x}_{1} \text { for } \mathbf{x}_{2} \\
\text { Solve } \mathbf{L}_{\varphi \varphi}^{T} \cdot \mathbf{x}_{1}=\mathbf{x}_{2} \text { for } \mathbf{x}_{1} \\
\mathbf{v}_{1}:=\mathbf{K}_{u \varphi} \cdot \mathbf{x}_{1} \\
\mathbf{v}_{2}:=\mathbf{A}_{\sigma u t} \cdot \mathbf{U} \\
\mathbf{v}_{1}:=\mathbf{v}_{1}+\mathbf{v}_{2} \\
\text { Solve } \mathbf{L}_{M} \cdot \mathbf{v}_{2}=\mathbf{v}_{1} \text { for } \mathbf{v}_{2} \\
\text { Solve } \mathbf{L}_{M}^{T} \cdot \mathbf{z}=\mathbf{v}_{2} \text { for } \mathbf{z}
\end{gathered}
$$

To solve problem $B$ we need a procedure for calculating the vector $\mathbf{z}=\mathrm{A}_{\mathrm{M}}^{-1} \cdot \mathbf{U}$ or $\mathbf{z}=\mathbf{A}_{\sigma}^{-1} \cdot \nu$; $v=\mathbf{M}_{u l} \cdot \mathbf{U}$ for the iterated vectors $\mathbf{U}$. Hence, here, to determine the vector $\mathbf{z}$, we require to solve the system of linear algebraic equations 


$$
\mathbf{G}_{\sigma}=\mathbf{K}-\sigma \mathbf{M}=\left\|\begin{array}{ll}
\mathbf{A}_{\sigma u ! \prime} & \mathbf{K}_{u \varphi} \\
\mathbf{K}_{u \varphi}^{T} & -\mathbf{K}_{\varphi \varphi}
\end{array}\right\|, \mathbf{G}_{\sigma} \cdot\left\{\begin{array}{l}
\mathbf{z} \\
\mathbf{x}
\end{array}\right\}=\left\{\begin{array}{l}
\mathbf{v} \\
0
\end{array}\right\}
$$

for the vector $\lfloor z, x\rfloor^{T}$, or the system of linear algebraic equations

$$
A_{\sigma} \cdot z=v
$$

for the vector $\mathbf{z}$.

System of linear algebraic equations (1.17) can be solved using $L D L^{T}$-factorization of the matrix $\mathbf{G}_{\sigma}$ and subsequent separation of the vector $\mathbf{z}$ from the vector of the solutions. This method does not require the formation of blocks of the matrix $\mathbf{K}$. An alternative approach is to use iteration methods to solve system of linear algebraic equations (1.17) or (1.18), for which multiplications of $\mathbf{G}_{\sigma}$ or $\mathbf{A}_{\boldsymbol{\sigma}}$ by vectors are required. This operation, in the block approach for system of linear algebraic equations (1.18), is carried out by the first six steps of algorithm (1.16).

\section{MODE EXPANSION METHODS FOR A DYNAMIC ANALYSIS OF PIEZOELECTRIC DEVICES}

The classical method of expansion in eigenforms or modes for solving linear problems of steady oscillations and non-stationary problems, as they apply to the analysis of piezoelectric devices, has a singularity, due to the presence of an uncoupled component of the electric potential $\varphi$ [5].

As in Section 1, we will investigate piezoelectric devices ignoring temperature factors and acoustic media, but we will now include the damping properties in the consideration. Like the approximate method of describing the Rayleigh damping mechanism, used in structural analysis, for piezoelectrics we will assume

$$
\mathbf{C}_{u t \prime}=\alpha_{d} \mathbf{M}_{m i t}+\bar{\beta}_{d} \overline{\mathbf{K}}_{w \prime \prime}
$$

where $\alpha_{d}, \bar{\beta}_{d}$ are damping factors. Note that since the matrix $\overline{\mathbf{K}}_{u u}$ rather than $\mathbf{K}_{u u}$ occurs in $\mathbf{C}_{u u}$, formula (2.1) takes into account the totality of mechanical, piezoelectric and dielectric losses.

We will first consider the problem of the steady oscillations of piezoelectric devices. In the finiteelement equation (0.5), the matrices $\mathbf{M}$ and $\mathbf{K}$ and the vector a have the form (1.2), while

$$
\mathbf{C}=\left\|\begin{array}{ll}
\mathbf{C}_{u t \prime} & 0 \\
0 & 0
\end{array}\right\|, \mathbf{F}=\left\{\begin{array}{l}
\mathbf{F}_{t \prime}-\mathbf{K}_{u v} \cdot \mathbf{V} \\
\mathbf{F}_{\varphi}+\mathbf{K}_{\varphi \nu} \cdot \mathbf{V}
\end{array}\right\}
$$

where the vector $\mathbf{V}$ takes into account in $\mathbf{F}$ the non-uniform principal electric boundary conditions, while the principal mechanical boundary conditions are assumed to be zero.

Solving Eq. (0.5) for $\boldsymbol{\Phi}$, taking (1.2) and (2.2) into account, we obtain

$$
\begin{gathered}
\left(-\omega^{2} \mathbf{M}_{u t u}+j \omega \mathbf{C}_{\imath u t}+\overline{\mathbf{K}}_{u t t}\right) \cdot \mathbf{U}=\overline{\mathbf{F}}_{u t} \\
\mathbf{\Phi}=\mathbf{K}_{\varphi \varphi}^{-1} \cdot \mathbf{K}_{u \varphi}^{T} \cdot \mathbf{U}+\boldsymbol{\Phi}_{s t} \\
\overline{\mathbf{F}}_{u}=\mathbf{F}_{u}-\mathbf{K}_{u v} \cdot \mathbf{V}-\mathbf{K}_{u \varphi} \cdot \boldsymbol{\Phi}_{v t}, \boldsymbol{\Phi}_{s t}=-\mathbf{K}_{\varphi \varphi}^{-1} \cdot\left(\mathbf{F}_{\varphi}+\mathbf{K}_{\varphi v} \cdot \mathbf{V}\right)
\end{gathered}
$$

The vector $\boldsymbol{\Phi}_{s t}$ from (2.5) is also an uncoupled component of the electric potential, determined, in fact, from the separate problem of quasi-electrostatics

$$
\mathbf{K}_{\varphi v p} \cdot \boldsymbol{\Phi}_{s t}=-\mathbf{F}_{\varphi}-\mathbf{K}_{\varphi \nu} \cdot \mathbf{V}
$$

We will find the solution of problem (2.3) in the form of an expansion in eigenvectors (modes) $\mathbf{W}_{\gamma m}$ of problem (1.5), (1.6) and the same homogeneous principal boundary conditions

$$
\mathbf{U}=\sum_{m=1}^{n} Z_{m} \mathbf{W}_{\gamma m}
$$


Here $\gamma=r$ or $\gamma=$ a depending on the correspondence of the homogeneous principal boundary conditions of problem (2.3)-(2.5) and (1.5), (1.6), where usually $\gamma=r$.

Substituting (2.7) into (2.3) and multiplying the equation obtained scalarly by $\mathbf{W}_{\gamma k}^{T}$, and taking into account the orthogonality relations (1.7) and (1.8) and representation (2.1) we obtain

$$
Z_{k}=\frac{1}{\omega_{k}^{2}-\omega^{2}+2 j \xi_{k} \omega \omega_{k}} P_{k}
$$

where

$$
P_{k}=\mathbf{W}_{\gamma k}^{r} \cdot \overline{\mathbf{F}}_{u}, \quad \xi_{k}=\alpha_{d} \frac{1}{2 \omega_{k}}+\bar{\beta}_{d} \frac{\omega_{k}}{2}
$$

The solutions of the problems of the steady oscillations of piezoelectric devices by the method of expansion in oscillation modes are also determined by formulae (2.7)-(2.9) and (2.4).

The solutions of non-stationary problems $(0.1),(1.2),(2.2),(2.1)$ by the mode-expansion method were found in a similar way, only now the coefficients $Z_{m}$ in (2.7) are functions of time, like the components of the vector $F$ of the right-hand sides. The final expressions for the coefficients $Z_{k}$ have the same form as in the structural analysis [6].

The advantages and disadvantages of the mode-expansion method are well known from experience in solving problems of structural analysis. Thus, an important advantage of the method is the possibility of the direct determination of the damping factors $\xi_{k}$ of the individual modes, without taking the formulae from (2.9) into account. These factors can be specified from the experimentally measured values of the mechanical $Q$-factor $Q_{k}$ of the modes with number $k: \xi_{k}=1 /\left(2 Q_{k}\right)$.

The presence of the uncoupled potential $\Phi_{s t}$ and the matrices $\mathbf{K}_{u \varphi}$, in (2.4) and (2.5) makes it necessary to use partitioned forms of the matrix $\mathbf{K}$ even if, when finding the eigenvalues and the modes, this approach has also not been used. Note that by specifying the vectors of the external actions $\mathbf{F}_{u}, \mathbf{F}_{\varphi}$ and $\mathbf{V}$ in advance and permitting only a proportional change in them later, one can calculate the quantities $P_{k}$ in (2.9) at the stage of solving the eigenvalue problem.

\section{EXPLICIT-IMPLICIT SCHEMES FOR INTEGRATING THE EQUATIONS OF ACOUSTOELECTROELASTICITY WITH RESPECT TO TIME FOR UNSTEADY PROCESSES}

We will consider non-stationary problems of acousto electroelasticity without temperature effects. As in Section 2, expressing the electric potential $\Phi$ in terms of $\mathbf{U}$, we can convert system $(0.1)-(0.3)$ without temperature fields $\mathbf{T}$ and the dissipative properties of the acoustic medium $\left(\widetilde{\mathbf{R}}_{\imath \psi}=0\right.$ [2]) to the form

$$
\begin{gathered}
\mathbf{M} \cdot \ddot{\mathbf{a}}+\mathbf{C} \cdot \dot{\mathbf{a}}+\mathbf{K} \cdot \mathbf{a}=\mathbf{F} \\
\boldsymbol{\Phi}=\mathbf{K}_{\varphi \varphi}^{-1} \cdot \mathbf{K}_{t \varphi}^{T} \cdot \mathbf{U}+\boldsymbol{\Phi}_{s t}, \quad \boldsymbol{\Phi}_{s t}=-\mathbf{K}_{\varphi \varphi}^{-1} \cdot\left(\mathbf{F}_{\varphi}+\mathbf{K}_{\varphi v} \cdot \mathbf{V}\right)
\end{gathered}
$$

where

$$
\begin{gathered}
\mathbf{M}=\left\|\begin{array}{ll}
\mathbf{M}_{u \prime \prime} & 0 \\
0 & \mathbf{M}_{\psi \psi}
\end{array}\right\|, \mathbf{C}=\left\|\begin{array}{ll}
\mathbf{C}_{u \prime \prime} & \mathbf{R}_{u \psi} \\
-\mathbf{R}_{u \psi}^{T} & \mathbf{C}_{\psi \psi}
\end{array}\right\|, \mathbf{K}=\left\|\begin{array}{ll}
\overline{\mathbf{K}}_{u u} & 0 \\
0 & \mathbf{K}_{\psi \psi}
\end{array}\right\| \\
\mathbf{F}=\left\lfloor\mathbf{F}_{u}-\mathbf{K}_{u \nu} \cdot \mathbf{V}-\mathbf{K}_{u \psi \varphi} \cdot \mathbf{\Phi} .0\right]^{T}, \mathbf{a}=\lfloor\mathbf{U}, \boldsymbol{\Psi}\rfloor^{T}
\end{gathered}
$$

and, in system (3.1), (3.2), one can also take into account the non-uniform principal mechanical boundary conditions.

To integrate problem (3.1)-(3.4) with respect to time it is convenient to use the family of Wilson and Newmark schemes, presented in unified form [7]. A special case of the schemes from [7] is the usual method of central differences, which will also be considered further.

In the time net $t_{i}=\tau_{i}(i=0,1,2, \ldots ; \tau=\Delta t$ is the time step) we will approximate the derivatives with respect to time by the central differences 


$$
\ddot{\mathbf{a}}_{i}=\frac{1}{\tau^{2}}\left(\mathbf{a}_{i+1}-2 \mathbf{a}_{i}+\mathbf{a}_{i-1}\right), \dot{\mathbf{a}}_{i}=\frac{1}{2 \tau}\left(\mathbf{a}_{i+1}-\mathbf{a}_{i-1}\right)
$$

where $\mathbf{a}_{i}=\mathbf{a}\left(t_{i}\right)$.

Substituting (3.5) into (3.1) and (3.2), specified for the instant of time $t_{i}$, we obtain a step scheme of the form.

$$
\begin{gathered}
\mathbf{M}^{e f f} \cdot \mathbf{a}_{i+1}=\mathbf{F}^{c f f}\left(\mathbf{F}_{i i}, \mathbf{V}_{i}, \mathbf{\Phi}_{i}, \mathbf{a}_{i}, \mathbf{a}_{i-1}\right), \quad \mathbf{M}^{e f f}=\frac{1}{\tau^{2}} \mathbf{M}+\frac{1}{2 \tau} \mathbf{C} \\
\mathbf{F}^{c t f}=\mathbf{F}_{i}+\left(\frac{2}{\tau^{2}} \mathbf{M}-\mathbf{K}\right) \cdot \mathbf{a}_{i}-\left(\frac{1}{\tau^{2}} \mathbf{M}-\frac{1}{2 \tau} \mathbf{C}\right) \cdot \mathbf{a}_{i-1}
\end{gathered}
$$

After determining $\mathbf{a}_{i+1}=\left\lfloor\mathbf{U}_{i+1}, \boldsymbol{\Psi}_{i+1}\right\rfloor^{T}$ from (3.6), (3.7) and (3.4) from the quantities $\mathbf{F}_{u i}, \mathbf{V}_{i}, \Phi_{i}, \mathbf{a}_{i}$, $\mathbf{a}_{i-1}$ to change to the next time layer one needs to determine $\Phi_{i+1}$ from (3.2), i.e. solve the system of linear algebraic equations

$$
\mathbf{K}_{\varphi \varphi} \cdot \boldsymbol{\Phi}_{i+1}=\mathbf{K}_{u \varphi \varphi}^{T} \cdot \mathbf{U}_{i+1}-\mathbf{F}_{\varphi, i+1}-\mathbf{K}_{\varphi v} \cdot \mathbf{V}_{i+1}
$$

Hence, even if it is possible to diagonalize the matrix $\mathbf{M}^{\text {eff }}$ and the scheme in step (3.6) becomes explicit, at the stage of finding $\Phi_{i+1}$ it is necessary to solve system of linear algebraic equations (3.8). Scheme (3.6)-(3.8) is similar to that described earlier [8] and, for the reasons mentioned, was called an explicitimplicit integration scheme. Naturally, scheme (3.6)-(3.8) is conditionally stable, like the usual method of central differences.

We will discuss separately the problems related to finding the reciprocal matrix $\left(\mathbf{M}^{\text {eff }}\right)^{-1}$. From (3.6) and (3.3) we have

$$
\mathbf{M}^{\prime \prime \prime}=\frac{1}{2 \tau}\left\|\begin{array}{cc}
\frac{2}{\tau} \mathbf{M}_{u m}+\mathbf{C}_{m t} & \mathbf{R}_{u \psi} \\
-\mathbf{R}_{u \psi}^{T} & \frac{2}{\tau} \mathbf{M}_{\psi \psi}+\mathbf{C}_{\psi \psi}
\end{array}\right\|
$$

Here $\mathbf{M}_{u u}$ and $\mathbf{M}_{\psi \psi}$ are the matrices of the "masses" of the solid-state structure and the acoustic medium respectively, $\mathbf{C}_{4 \psi}$ is a matrix obtained as a result of finite-element approximation of the impedance conditions on the specified outer boundaries of the acoustic medium and $\mathbf{R}_{u \psi}$ is the coupling matrix of the solid-state structure and the acoustic medium along their outer boundary $\Gamma_{h w s}$ [2], where it is convenient to relate the normal components of the displacements of the solid-state structure on $\Gamma_{\text {hws }}$ at common nodes to the velocity potentials of the acoustic medium. If we assume that the damping matrix $\mathbf{C}_{u u}$ is proportional to the matrix of the masses $\mathbf{M}_{t u}\left(\mathbf{M}_{u u}=\alpha_{d} \mathbf{C}_{u u}\right)$, all the element matrices of the blocks of the matrices from (3.9) can be calculated as integrals solely of the products of form functions. At the same time, the element matrices of the blocks of matrix $\mathbf{K}$ are calculated as integrals of products of the derivatives of form functions. Consequently, standard approaches, similar to methods of diagonalizing a mass matrix in structural analysis can be applied to the element matrices of the blocks from (3.9).

We will calculate the element matrices for the blocks of matrices (3.9) by the method of nodal integration [9]. Then, the matrices $\mathbf{M}_{u u}, \mathbf{C}_{u u}, \mathbf{M}_{\psi \psi}$ and $\mathbf{C}_{\psi \psi}$ become diagonal. In the element matrices $\mathbf{R}_{u \psi}$, with the appropriate numbering of the degrees of freedom, the non-zero elements will be found only on the second principal diagonal, opposite the usual matrix diagonal. Finally, after renumbering the degrees of freedom, the matrix $\mathbf{M}^{\text {eff }}$ can be represented in the following partitioned-diagonal form

$$
\mathbf{M}^{(\prime \prime} \rightarrow \mathbf{M}_{d}^{\prime \prime f}=\left\|\begin{array}{cc}
\mathbf{D}_{M} & 0 \\
0 & \mathbf{B}_{M}
\end{array}\right\|
$$

where $\mathbf{D}_{M}$ is the diagonal submatrix assembled from the contributions of the degrees of freedom, which do not interact with the boundary $\Gamma_{h w s}$ and $\mathbf{B}_{M}$ is a partitioned-diagonal submatrix, each block of which with number $j$ corresponds to the interaction of the solid-state structure and the acoustic medium at one node on $\Gamma_{h w s}$ and is represented by the $2 \times 2$ matrix 


$$
\mathbf{B}_{M j}=\left\|\begin{array}{cc}
b_{11}^{j} & b_{12}^{j} \\
-b_{12}^{j} & b_{22}^{j}
\end{array}\right\|
$$

where the component $b_{11}^{j}$ is the contribution of the matrix $\mathbf{M}_{u u}, b_{22}^{j}$ is the contribution of the matrix $\mathbf{M}_{\psi \psi}$, and $b_{12}^{j}$ is the contribution of the matrix $\mathbf{R}_{u \psi}$.

The reciprocal of the matrix $\mathbf{M}_{d}^{\text {eff }}$ is easily calculated in explicit form

$$
\left(\mathbf{M}_{d}^{(\prime \prime l}\right)^{-1}=\left\|\begin{array}{cc}
\mathbf{D}_{M}^{-1} & 0 \\
0 & \mathbf{B}_{M}^{-1}
\end{array}\right\| \quad \mathbf{B}_{M j}^{-1}=\frac{1}{b_{11}^{j} b_{22}^{j}+\left(b_{12}^{j}\right)^{2}}\left\|\begin{array}{cc}
b_{22}^{j} & -b_{12}^{j} \\
b_{12}^{j} & b_{11}^{j}
\end{array}\right\|
$$

Hence, stages (3.6) and (3.7) of the step scheme can be represented in the explicit form

$$
\mathbf{a}_{i+1}=\left(\mathbf{M}_{d}^{e f f}\right)^{-1} \cdot\left[\mathbf{F}_{i}+\left(\frac{2}{\tau^{2}} \mathbf{M}_{d}-\mathbf{K}\right) \cdot \mathbf{a}_{i}-\left(\frac{1}{\tau^{2}} \mathbf{M}_{d}-\frac{1}{2 \tau} \mathbf{C}_{d l}\right) \cdot \mathbf{a}_{i-1}\right]
$$

where the matrices $\mathbf{M}_{d}$ and $\mathbf{C}_{d}$ are diagonalized to the partitioned form in the same way as the matrix $\mathbf{M}_{d}^{e f f}$.

Note that in the block diagonalization of the matrices $\mathbf{M}^{\text {eff }}, \mathbf{M}$ and $\mathbf{C}$ one can also use the method of separating the "principal diagonal" [10], assuming that the second principal diagonal can be separated for $\mathbf{R}_{u \psi}$.

As a result, we have an explicit-implicit integration scheme (3.10), (3.8) using the method of central differences, for which procedures of multiplying a matrix by a vector and solving system of linear algebraic equations (3.8) are required.

\section{PYROELECTRIC DEVICES AT LOW MODULATION FREQUENCIES OF THE TEMPERATURE FIELDS}

It was pointed out in [11] that the operation of puroelectric pickups at low modulation frequencies of the temperature fields can be modelled following from $(0.1)-(0.3)$ by a system of finite-element methods (the effect of the acoustic medium in this case is ignored)

$$
\begin{gathered}
\mathbf{C}_{\theta \theta} \cdot \dot{\mathbf{T}}+\mathbf{K}_{\theta \theta} \cdot \mathbf{T}=\mathbf{F}_{\theta} \\
\mathbf{K}_{m !} \cdot \mathbf{U}+\mathbf{K}_{u \varphi} \cdot \boldsymbol{\Phi}=\mathbf{F}_{\|}+\mathbf{K}_{u \theta} \cdot \mathbf{T} \\
\mathbf{K}_{\| \varphi}^{T} \cdot \mathbf{U}-\mathbf{K}_{\varphi \varphi} \cdot \mathbf{\Phi}=\mathbf{F}_{\varphi}-\mathbf{K}_{\varphi \theta \theta} \cdot \mathbf{T}
\end{gathered}
$$

Problem (4.1)-(4.3) turns out to be connected with respect to the temperature fields and the displacements-electric potentials only via the vectors on the right-hand sides. Here it is convenient to form individual blocks $\mathbf{C}_{\theta \theta}, \mathbf{K}_{\theta \theta}, \mathbf{K}_{u \theta}$ and $\mathbf{K}_{\varphi \theta}$. System (4.1)-(4.3) can be solved by standard methods, both for problems of harmonic modes and for non-stationary problems.

\section{NUMERICAL EXPERIMENTS}

When solving the generalized eigenvalue problem (1.5) from Section 1 the ARPACK software package [12], which uses Arnoldi's method, was employed. We chose the ARPACK program for the eigenvalue problem in the form (1.9). All the procedures for introducing the parameters of the initial problem, forming the finite-element model and outputting the results, were carried out using the ACELAN finite-element program [13]. The ACELAN software package was specially supplemented with programs which carry out the steps of algorithm (1.14)-(1.16) with partitioned matrices, which were stored in a sparse row format. The code which generated the ARPACK package was included in the library of the ACELAN subroutines.

As an example, we will consider a composite piezoelectric radiator of acoustic waves of axisymmetrical form, a meridian section of which is shown in Fig. 1 in a cylindrical system of coordinates Orz. Mechanical oscillations in the device are generated by a piezoelectric element of radius $r_{1}$ and thickness $h_{6}$, made of TsTBS- 3 thickness- 


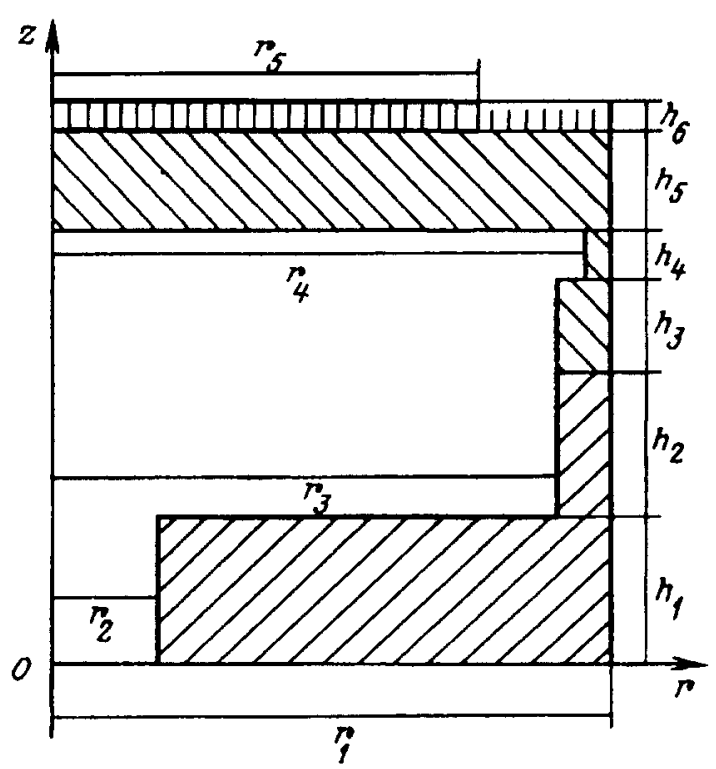

Fig. 1

polarized piezoelectric ceramics (shown by the vertical hatching in Fig. 1). The end surfaces of the piezoelectric element $z=H_{1}=h_{1}+\ldots+h_{5}$ and $z=H_{2}=h_{1}+\ldots+h_{6}$ are coated with continuous circular electrodes of the same radius $r_{5}$. The piezoelectric element is rigidly clamped to a hollow steel cylinder with an upper cap (represented by the left-leaning hatching) which in turn is rigidly clamped to a hollow cylinder of aluminium, the bottom end of which has a circular opening (represented by the right-leaning hatching). To increase the compliance for flexural oscillations, the steel part of the device has a recess $h_{4}$ thick with a dimension $\delta=r_{4}-r_{2}$.

The geometrical dimensions shown in Fig. 1 are as follows: $r_{1}=21 \mathrm{~mm}, r_{2}=4 \mathrm{~mm}, r_{3}=19 \mathrm{~mm}$, $r_{4}=20 \mathrm{~mm}, r_{5}=16 \mathrm{~mm}, h_{1}=h_{2}=5.5 \mathrm{~mm}, h_{3}=3.5 \mathrm{~mm}, h_{4}=2 \mathrm{~mm}, h_{5}=3.5 \mathrm{~mm}$ and $h_{6}=1.2 \mathrm{~mm}$. The material constants of the TsTBS-3 piezoelectric ceramics were taken from [14], the steel has a density $\rho_{s t}=7.86 \times 10^{3} \mathrm{~kg} / \mathrm{m}_{3}$, Young's modulus $E_{s t}=2 \times 10^{11} \mathrm{~N} / \mathrm{m}^{2}$ and Poisson's ratio $\nu_{s t}=0.29$, while the aluminium has the following constants: $\rho_{a l}=2.7 \times 10^{3} \mathrm{~kg} / \mathrm{m}^{3}, E_{a l}=7.1 \times 10^{10} \mathrm{~N} / \mathrm{m}^{2}$ and $\nu_{a l}=0.33$.

For this device it is required to determine the first frequencies of flexural oscillations $f_{r(j)}$, excited by the potential difference

$$
\left.\varphi\right|_{z=H_{1}}-\left.\varphi\right|_{z=H_{2}}=V \exp \left(j 2 \pi f_{r(j)} t\right)
$$

between the electrodes of the piezoelectric element. To do this we constructed finite-element models and we solved eigenvalue problems (1.1)-(1.3) to determine the electric resonance frequencies $f_{r k}$ and (1.1), (1.2) and (1.4) for the electric antiresonance frequencies $f_{a m}$. These two problems, with homogeneous natural mechanical boundary conditions, differ solely in the electrical boundary conditions; for $f_{r k}$ we use $\varphi=0$ when $z=H_{1}$ and $z=H_{2}$, while for $f_{a m}$

$$
\varphi=\left\{\begin{array}{cc}
0 & \text { for } z=H_{1} \\
\text { const } \cdot \exp (j 2 \pi f t) & \text { for } z=H_{2}
\end{array} ; \int_{0}^{r_{5}} D_{2} r d r=0 \text { for } z=H_{2}\right.
$$

where $\mathbf{D}$ is the electric induction vector. By comparing the natural frequencies $f_{r k}$ and $f_{a m}$ obtained we find the actual working frequencies of electrical resonances $f_{r(j)}$ and $f_{a(j)}$, which are close to one another, but nevertheless differ considerably. The numbering $(j)$ of these frequencies, generally speaking, is not the same as the numberings $k$ and $m$.

The numerical results obtained in ACELAN using axisymmetrical quadratic triangular finite elements with six nodes, were compared with similar results obtained in ANSYS, where, for axisymmetrical problems of piezoelectric analysis, only four-angle finite elements with four nodes are possible. Below we present values of the natural frequencies of a piezoelectric radiator (in $\mathrm{RHz}$ ), calculated ACELAN with ND $=239$ and (in parentheses) by ANSYS with ND $=253$, where ND is the number of nodes in the finite-element models

$$
\begin{array}{ll}
f_{r 1}=0.1 ! \times 10^{-3}(0), & f_{a 1}=0.43 \times 10^{-3}(0), \\
f_{r 2}=14.55(14.44), & f_{a 2}=14.94(14.85), \\
f_{r \cdot 3}=22.11(22.01), & f_{a 3}=22.31(22.20) .
\end{array}
$$


As can be seen, there is quite good agreement between the results obtained using ACELAN and ANSYS.

The working frequencies of a piezoelectric radiator are the electrical resonance frequencies $f_{r(1)}=f_{r 2}$ or $f_{r(2)}=f_{r 3}$, for which the corresponding electrical antiresonance frequencies are $f_{a(1)}=f_{a 2}$ or $f_{a(2)}=f_{a 3}$. At these frequencies flexural oscillations of the piezoelectric radiator occur. As a result of calculations we obtained that the mode of oscillation at a frequency $f_{r(1)}$ contains pronounced antiphase motions of the lower and upper ends of the piezoelectric radiator, while the mode of oscillation at a frequency $f_{r(2)}$, on the other hand, contains in-phase motions. Note that the forms of the modes in calculations using ACELAN and ANSYS hardly differ from one another (Interesting practical applications of these results and the optimization of the dimensions of the piezoelectric radiator are outside the scope of this paper.)

In other test calculations in a modal analysis of electroelasticity problems we also obtained good agreement between the ACELAN and ANSYS results.

We would expect the mode-expansion method from Section 2 to be effective for analysing devices operating at pronounced resonance frequencies. For example, consider a longitudinal-longitudinal piezoelectric transformer [15], shown in Fig. 2. The sections of the transformer consist of TsTBS-3 piezoelectric ceramics [14] and have the following parameters $l_{1}=l_{2}=0.05 \mathrm{~m}, a=0.02 \mathrm{~m}$ and $b=0.005 \mathrm{~m}$. (The directions of polarization of the piezoelectric ceramics in the exciter and generator sections are shown by the bold arrows in Fig. 2.)

Under working conditions the electrode $x=l_{1}$ is grounded $(\varphi=0)$, while a potential $\varphi=V_{i n} \exp (j 2 \pi f t)$ is applied to the electrode $x=0$. The value of the potential on the electrode $\varphi=l_{1}+l_{2}$ is unknown, but the overall charge is zero. During operation the transformer generates a potential $\varphi=V_{\text {ou }} \exp (j 2 \pi f t)$ on the electrode $\varphi=I_{1}+l_{2}$ where $V_{\text {out }} \in C$. A measure of the efficiency of this device is the voltage transformation factor $K=\left|V_{\text {out }}\right| / V_{\text {in }}$. The fundamental operating frequencies of a longitudinal-longitudinal piezoelectric transformer are the first frequencies of electric resonance with tension-compression modes with respect to $x$.

We will compare the following models of a piezoelectric transformer: a model one-dimensionally elastic along $x$ with additional elements which describe the piezoelectric effects (1D), a model that is two-dimensionally piezoelectric in the Oxy plane using the hypothesis of a plane stressed state (2D) and a three-dimensional piezoelectric model (3D). In Table 1 we show the first electric resonance frequencies $f_{r(k)}$ and antiresonance frequencies $f_{a(k)}$ (in $\mathrm{kHz}$ ), in which piezoelectric couplings occur. (Here the number of working electrical resonance frequencies is not the same as their order numbers of the natural frequencies of the body considered.) The calculations were made in ANSYS with rectangular finite elements with a splitting of the length $l_{1}+l_{2}$ into 20 elements (1D, 2D and 3D), a width a in five elements (2D and 3D) and a thickness $b$ in two elements (3D).

It can be seen from Table 1 that the first tension-compression frequencies are well determined by the onedimensional theory.

We determined the transformation factor $\mathrm{K}$ from the solution of the problems of the steady-state oscillations for the one-dimensional model employing the method of expansion in four modes of oscillation both for the onedimensional and two-dimensional models with the solution of the complete system of linear algebraic equations (2.3).

We specified the damping for the one-dimensional model, using the mode-expansion method, via the attenuation factor $\xi_{k}=1 /(2 Q)$ with a mechanical Q-factor $Q=350$. For the one-dimensional and two-dimensional models, by solving the complete system of linear algebraic equations we will determine the attenuation in terms of the coefficients $\alpha_{d}$ and $\beta_{d}$, which occur in the representation of the damping matrix [4]

$$
\mathbf{C}_{m ! t}=\alpha_{d l} \mathbf{M}_{u t !}+\beta_{d} \mathbf{K}_{t u t}
$$

We will assume that the Q-factor of the piezoelectric transformer at resonance frequencies $f_{r(1)}$ and $f_{r(2)}$ are $Q_{(1)}$ and $Q_{(2)}$ respectively. By comparing representations (5.1) and (2.1) we an obtain the following approximate formulae

$$
\begin{aligned}
& \alpha_{d l}=2 \pi f_{r(1)} f_{r(2)}\left[\left(1+k_{(1)}^{2}\right) f_{r(2)} Q_{(2)}-\left(1+k_{(2)}^{2}\right) f_{r(1)} Q_{(1)}\right] / \Delta \\
& \beta_{d}=\left(1+k_{(1)}^{2}\right)\left(1+k_{2}^{2}\right)\left[f_{r(2)} Q_{(2)}-f_{r(1)} Q_{(1)}\right] /(2 \pi \Delta) \\
& \Delta=Q_{(1)} Q_{(2)}\left[\left(1+k_{(1)}^{2}\right) f_{r(2)}^{2}-\left(1+k_{(2)}^{2}\right) f_{r(1)}^{2}\right] \\
& k_{(1)}^{2}=\left(\mathbf{W}_{r(j)}^{T} \cdot \mathbf{K}_{\varphi(t)}^{r} \cdot \mathbf{K}_{\varphi(\varphi)}^{-1} \cdot \mathbf{K}_{\varphi(1)} \cdot \mathbf{W}_{r(j)}\right) /\left(\mathbf{W}_{r(j)}^{T} \cdot \mathbf{K}_{m+1} \cdot \mathbf{W}_{r(j)}\right)
\end{aligned}
$$

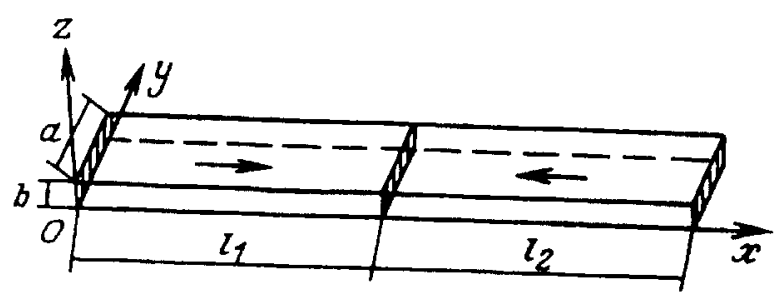

Fig. 2 
Table 1

\begin{tabular}{c|c|c|c|c}
\hline Model & $f_{r(1)}$ & $f_{a(1)}$ & $f_{r(2)}$ & $f_{a(2)}$ \\
\hline ID & 18.80 & 21.08 & 38.87 & 42.28 \\
2D & 18.70 & 20.87 & 38.39 & 41.66 \\
3D & 18.73 & 20.89 & 38.45 & 41.76 \\
\hline
\end{tabular}

Table 2

\begin{tabular}{l|l|l}
\hline Model & $K_{(1)}$ & $K_{(2)}$ \\
\hline ID (Mode sup.) & 65.92 & 66.21 \\
ID (Full) & 65.89 & 66.20 \\
2D (Full) & 63.97 & 64.97 \\
\hline
\end{tabular}

They were used to calculate the transformation factors $K_{j}$ in the two-dimensional model, while for the onedimensional model, the reduced system of linear algebraic equations which included the matrix $\overline{\mathbf{K}}_{u u}$, employed other values of the coefficients $\alpha_{d}$ and $\bar{\beta}_{d}$, formally obtained from (5.2) with $k_{(1)}=k_{(2)}=0$.

Table 2 shows the results of calculations of the transformation factors $K_{(j)}$ for the first two working frequencies, close to the first resonance frequencies $f_{r(j)}(j=1,2)$ from Table 1 . It can be seen that the mode-expansion method (Mode sup.) for this problem gives values of the transformation factors which hardly differ from the values calculated using methods which employ the solution of the complete system of linear algebraic equations (Full).

We wish to thank our colleagues O. N. Akopov, K. A. Nadolin and A. S. Skaliukh for developing the ACELAN software package, the results of the development of which were used to carry out the numerical experiments, and also Yu A. Kramarov for suggesting the problem of designing a piezoelectric radiator of original construction.

This research was partially supported by the Russian Foundation for Basic Research (99-01-01001).

\section{REFERENCES}

1. RAO, S. S., and SUNAR, M., Analysis of distributed thermopiezoelectric sensors and actuators in advanced intelligent structures. AIAA Joumal, 1993, 31, 7, 1280-1286.

2. NASEDKIN, A. V., The analysis, using the finite-element method, of piezoelectric transducers loaded by an acoustic medium. Izv. Vuzov. Sev-Kavkaz. Region Yestestv. Nauki, 1999, 1, 48-51.

3. BELOKON', A. V. and NASEDKIN, A. V., Some properties of the natural frequencies of electroelastic bodies of limited dimensions. Prikl. Mat. Mekh., 1996, 60, 1, 151-158.

4. ANSYS. Theory Reference. Rel. 5.5. Edited by P. Kothnke. ANSYS Inc. Houston, TX, 1998.

5. ZHARII, O. Yu., An eigenfunction expansion method in problems of dynamic electroelasticity. Prikl. Mat. Mekh., 1990, 54, $1,109-115$.

6. VOL'MIR, A. S., KURANOV, B. A. and TURBAIVSKII, A. T., The Statics and Dynamics of Complex Structures: Applied Multilevel Research Methods. Mashinostroyeniye, Moscow, 1989.

7. NASEDKIN, A. V., Alternative formations of Newmark's and Wilson's methods. In Present Problems in Continuum Mechanics. Proceedings of the Second intemational Conference. Rostov-on-Don, 1996. MP "Kniga", Rostov-on-Don, 1996, Vol. 2, 115-119.

8. WOJCIK, G. L., VAUGHAN, D. K., ABBOUD, N. MOULD, J. Electromechanical modeling using explicit time-domain finite elements. Proc. IEEE Ultrason. Symp., 1993, 2, 1107-1112.

9. FRIED, I., and MALKUS, D. S., Finite element mass matrix lumping by numerical integration with no convergence loss. Int. J. Solids and Structures, 1975, 11, 4, 461-466.

10. HINTON, E., ROCK, T., and ZIENKEWICZ, O. C. A note on mass lumping and relating processes in finite element method. Int. J. Earthquake. Eng. and Struct. Dyn., 1976, 4, 3, 245-249.

11. NASEDKIN, A. V., A finite-element dynamic analysis of piezoelectric acoustic-wave radiators. In: Present Problems of Continuum Mechanics. Proceedings of the 4th International Conference, Rostov-on-Don. Izd. Sev.-Kavkaz. Nauch. Tsentra Vysshikh Shkol, Rostov-on-Don, 1999, Vol. 2, 89-93.

12. LEHOUCQ, R. B., SORENSEN, D. C., VU, P. and YANG C. ARPACK: An implementation of the Implicitly Re-started Arnoldi Iteration that computes some of the eigenvalues and eigenvectors of a large sparse matrix. Available from netlib@ornl. gov under the directory scalapack, 1996.

13. AKOPOV, O. N., BELOKON', A. V., NADOLIN, K. A. et al., Statistical analysis of piezoelectric devices in ACELAN. I. Structure and possibilities II. Numerical experiments. In Present Problems of Continutum Mechanics. Proceedings of the 4th International Conference. Rostov-on-Don, 1998. Izd. Sev.-Kavk. Nauch Tsentra Vysshikh Shkol. Rostov-on-Don, 1999, Vol. 1, 14-22.

14. ARONOV, B. S., Piezoelectric Ceramic Electromechanical Transducers. Energoatomizdat, Leningrad, 1990.

15. LAVRINENKO, V. V., Piezoelectric Transformers. Energiya, Moscow, 1975. 